\title{
Feasibility and Postoperative Analgesia of Transversus Abdominis Plane Block in Patients Undergoing Total Abdominal Hysterectomy
}

\author{
Owono Etoundi Paul ${ }^{1,2 *}$, Bengono Bengono Roddy ${ }^{2}$, Tochie Joël ${ }^{2}$, Afané Ela Anatole ${ }^{1,2}$ and Ze Minkandé \\ Jacqueline ${ }^{2}$
}

${ }^{1}$ Department of Anaesthesiology, Yaoundé Central Hospital, Africa

${ }^{2}$ Department of Surgery and Specialities /Anaesthesiology, University of Yaoundé, Africa

Submission: October 29, 2017; Published: November 21, 2017

*Corresponding author: Owono Etoundi Paul, Department of Surgery and Specialities/Anaesthesiology University of Yaoundé, P.O. Box 1364 Yaoundé, Cameroon, Tel: 237-677-981-951, Email: owonop@gmail.com

Abstract

Background: The transversus abdominis plane (TAP) block is a regional anaesthetic technique that provides postoperative analgesia after abdominal surgery. This study was done to evaluate its feasibility and its efficacy on postoperative analgesia in patients undergoing total abdominal hysterectomy in a resource-limited setting.

Methods: Ninety women, aged 30 to 68 years, classified ASA I and II, proposed for total abdominal hysterectomy indicated for uterine fibroids, were divided into two groups by randomization. Group A received a blind bilateral TAP block with ropivacaïne (1.5 mg/kg on each side) and after installation of sensory block, the general anaesthesia was carried out with propofol, fentanyl, rocuronium and isoflurane. Group B received only general anaesthesia with the same protocol like in group A. The success rate of TAP block was recorded in group A. In both groups, postoperative pain scores at rest, coughing efforts and postoperative analgesic consumption have been compared.

Results: In Group A, the success rate of blind TAP block was $95.5 \%$. At the complete awakening of the patients, the mean visual analogue scale scores were significantly lower in patients in Group A compared to the patients in Group B (at rest $10.46 \pm 0.92$ versus $32.05 \pm 20.81 \mathrm{~mm}$, $p=0.006$ and with coughing $23.61 \pm 12.04$ versus $41.25 \pm 18.50 \mathrm{~mm}, p=0.009$ ). Compared to women in Group B, those in Group A had significantly lower visual analogue scale scores at rest, coughed less during the first 48 hours postoperatively and consumed significantly less ketoprofen (176.47 $\pm 65.40 \mathrm{mg}$ versus $300.00 \pm 41.40 \mathrm{mg}, \mathrm{p}=0.000)$ and no morphine $(0 \mathrm{mg}$ versus $15.19 \pm 3.6, \mathrm{p}=0.000$ ).

Conclusion: In a resource-limited setting, blind TAP block is feasible and should be practiced because it is easy to achieve. It significantly decreases postoperative pain and reduces the requirement for opioids and other analgesics.

Keywords: Transversus Abdominis Plane Block; Feasibility; Postoperative Analgesia; Total Abdominal Hysterectomy

\section{Introduction}

Total abdominal hysterectomy is associated with severe postoperative pain which may last 48 hours. This pain is acute, nociceptive and inflammatory with a profound hyperalgesic component [1,2]. Hence, a multimodal management of this postoperative pain cannot be overemphasized. Although not used in our country, the Transversus Abdominis Plane (TAP) block has proven its efficacy in the management of postoperative pain after total abdominal hysterectomy [3-6]. Thus, this study was conducted to assess the feasibility and efficacy of the TAP block in the management of postoperative pain after total abdominal hysterectomy. The research goal is to improve the quality of the management of postoperative pain in resourcechallenged settings.

\section{Patients and Methods}

After approval by the National Ethical Committee and obtaining a signed informed consent from participants, we carried out a prospective simple-blinded randomised study at the Anaesthesiology and Intensive Care Unit of the Yaoundé Central Hospital from January 01, 2013 to December 31, 2015. We enrolled ASA I and II patients who were to undergo total abdominal hysterectomy for uterine fibroids and who could understand the visual analogue scale (VAS). We excluded patients with obesity (body mass index $>30 \mathrm{~kg} / \mathrm{m} 2$ ), cardiovascular diseases, neuropsychiatric pathologies and those on longterm analgesics. Other exclusion criteria were the inability to comprehend the VAS, known allergy or a contra-indication to any 
of the drugs used, contra-indication to loco-regional anaesthesia and patients undergoing any concomitant surgery in addition to total abdominal hysterectomy. Patients were blindly and equally allocated to two groups from a table of randomization. In Group A, a bilateral TAP block was performed on conscious patients according to McDonnell blind technique.

Ropivacaïne $(1.5 \mathrm{mg} / \mathrm{kg}$ diluted in $20 \mathrm{ml}$ of normal saline) was administered on either side without exceeding a total dose of $225 \mathrm{mg}$. The sensory block was tested by skin pricks at the operative site every five minutes. Failure of the TAP block was considered in the setting of persistent pain after 30 minutes of realisation of the TAP block. Patients with failed blocks were excluded from Group A. The same Anaesthesiologist performed the TAP block. After installation of the sensory block, general anaesthesia was induced using propofol $(2.5 \mathrm{mg} / \mathrm{kg})$, fentanyl $(3 \mathrm{mcg} / \mathrm{kg})$ and rocuronium $(0.9 \mathrm{mg} / \mathrm{kg})$. In Group B, the TAP block was not performed. In this group, only general anaesthesia was performed using the same induction protocol described in Group A.

In both groups, orotracheal intubation was done. Isoflurane and re-injections of fentanyl $(1.5 \mu \mathrm{g} / \mathrm{kg})$ were used for maintenance of general anaesthesia. The intra-operative ventilation was mechanical. The same surgical team performed total abdominal hysterectomy through a Pfannenstiel incision. The management of postoperative pain began after surgery and at complete awakening of the patient (H0) by evaluating the intensity of the pain using the VAS graduated from 0 to $100 \mathrm{~mm}$. When the VAS was zero, between 1 and 3, 4 and 7 or greater than 7 , the patient received respectively: no analgesic, $1 \mathrm{~g}$ paracetamol slow intravenous (SIV), paracetamol $1 \mathrm{~g}$ and $100 \mathrm{mg}$ ketoprofen (SIV), paracetamol $1 \mathrm{~g}$ and ketoprofen $100 \mathrm{mg}$ (SIV) plus morphine in titrations at $2 \mathrm{mg}$ (SIV) every five minutes until the pain subsides.

The pain was evaluated every hour during four hours, then four hourly till the 48th hour, then at rest and during coughing efforts. The administration of analgesics in the postoperative period was based on the VAS, as well as the kinetics and intervals of administration of the drugs: paracetamol (1g/6hours), ketoprofen (100 mg/8 hours), morphine (titration/6 hours). Variables studied were: delay of onset of the sensitive block, the success rate of the TAP block in Group A; variation of the intensity of postoperative pain at rest, during coughing efforts, the postoperative consumption of analgesics in both groups. Statistical analysis was done using Epi Info 3.5.4 software. Numerical variables were reported as means and standard deviations. Categorical data were compared using the Fisher's exact test or the Pearson's Chi-square test where appropriate. A p-value less than 0.05 were considered significant.

\section{Results}

Eighty-eight women of ASA I $(n=40)$ and ASA II $(n=48)$, aged 30 to 68 years, were recruited for the study and their data was analyzed. A CONSORT flow diagram depicting the selection of participants through the trial is illustrated in Figure 1. The two groups were comparable in terms of age, body mass index, duration of surgery and anaesthesia (Table 1). In Group A, the mean duration for the realisation of the TAP block was $2.51 \pm$ 1.44 minutes. The mean duration of installation of the sensitive block was $16.24 \pm 3.12$ minutes. Two failed TAP block cases were observed, hence, a success rate of $95.5 \%$.

Table 1: Demographic and the operative characteristics in the two groups.

\begin{tabular}{|c|c|c|c|}
\hline Parameters & $\begin{array}{c}\text { Group A (n =43) } \\
\text { (Mean } \pm \text { SD) }\end{array}$ & $\begin{array}{c}\text { Group B (n= 45) } \\
\text { (Mean } \pm \text { SD) }\end{array}$ & p value \\
\hline Age (years) & $43.62 \pm 6.45$ & $44.41 \pm 6.61$ & 0.646 \\
\hline BMI (kg/m2) & $25.53 \pm 2.61$ & $26.18 \pm 2.11$ & 0.333 \\
\hline $\begin{array}{c}\text { Surgical time } \\
\text { (min) }\end{array}$ & $81.50 \pm 22.63$ & $89.97 \pm 18.01$ & 0.105 \\
\hline $\begin{array}{c}\text { Anaesthetic } \\
\text { time (min) }\end{array}$ & $102.55 \pm 27.67$ & $109.30 \pm 18.52$ & 0.148 \\
\hline
\end{tabular}

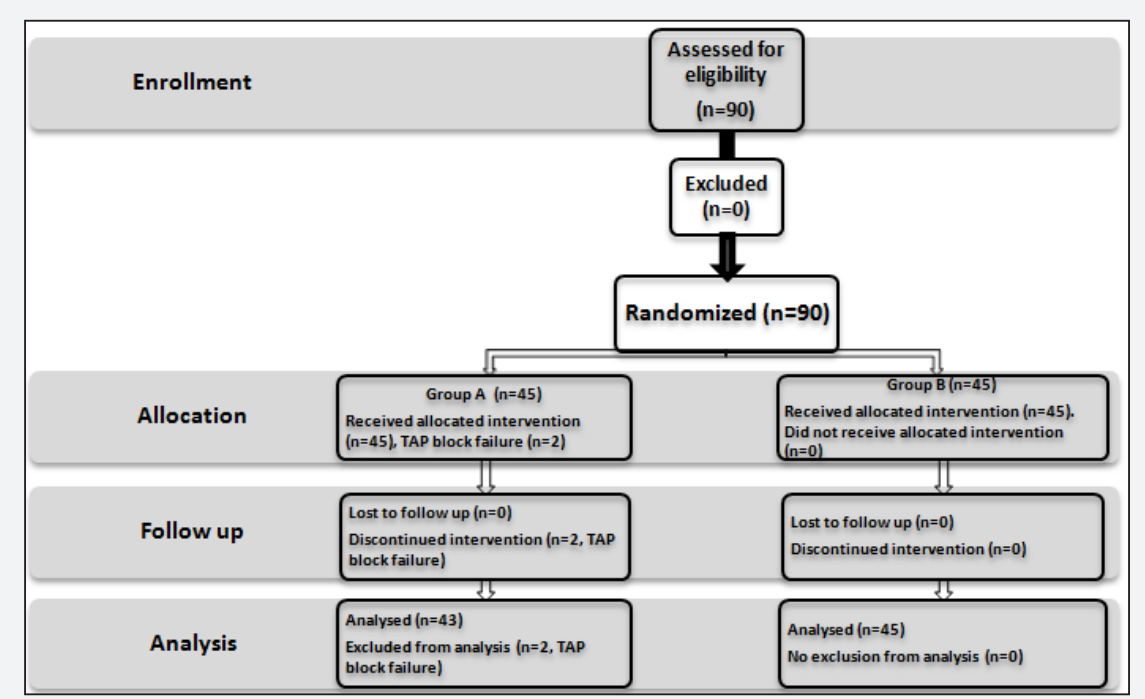

Figure 1: CONSORT Flow diagram for patient selection. 


\section{Journal of Anesthesia \& Intensive Care Medicine}

Regarding postoperative pain, the mean visual analogue scale scores (VAS) at the complete awakening of the patients (H0) were significantly lower in patients who received TAP block (Group A): at rest $10.46 \pm 0.92$ vs. $32.05 \pm 20.81 \mathrm{~mm}$, $\mathrm{p}=0.006$ and with coughing $23.61 \pm 12.04$ vs $41.25 \pm 18.50$ $\mathrm{mm}, \mathrm{p}=0.009$. During the first 48 hours postoperatively, there was a significant difference in the pain scores between the two groups. Women who did not receive TAP block (Group B) complained of significant pain at rest and on cough (Figures 2 \& 3). Postoperative analgesic consumption was higher in the Group B compared to the Group A, and morphine was not used in Group A (Table 2).

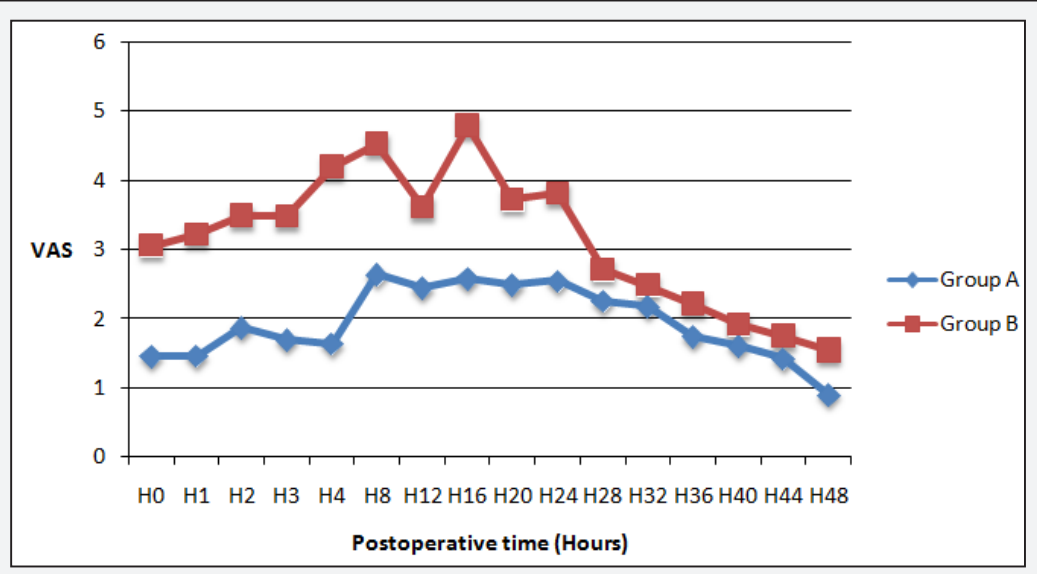

Figure 2 : Pain scores at rest. Visual Analogue scale (VAS) score corresponding $95 \%$ confidence intervals in the two groups

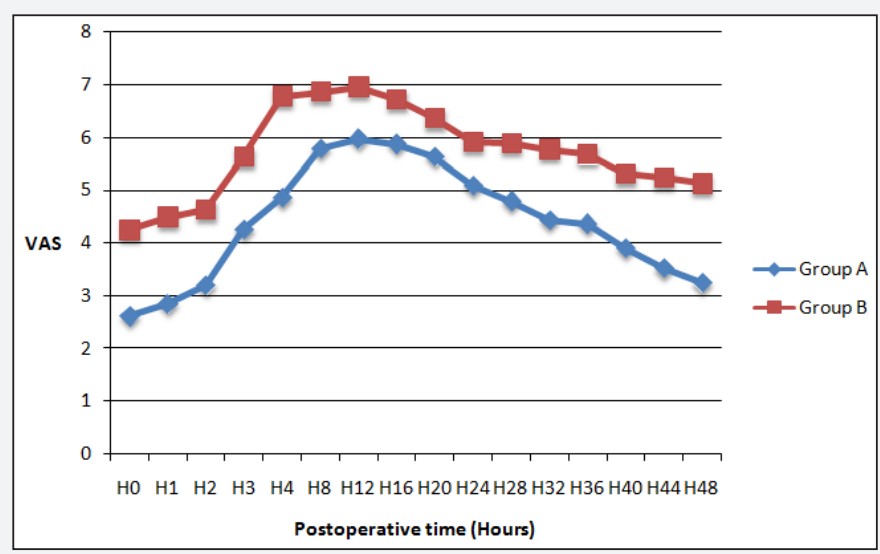

Figure 3 : Pain scores on coughing. Visual Analogue scale (VAS) score corresponding $95 \%$ confidence intervals in the two groups

Table 2 : Postoperative analgesic consumption.

\begin{tabular}{|c|c|c|c|}
\hline & $\begin{array}{c}\text { Group A(mean } \\
\pm \text { SD) }\end{array}$ & $\begin{array}{c}\text { Group B } \\
\text { (mean } \pm \text { SD) }\end{array}$ & p-value \\
\hline $\begin{array}{c}\text { Paracetamol } \\
\text { (g) }\end{array}$ & $7.73 \pm 0.51$ & $7.77 \pm 0.42$ & 0.846 \\
\hline $\begin{array}{c}\text { Ketoprofen } \\
\text { (mg) }\end{array}$ & $176.47 \pm 65.40$ & $\begin{array}{c}300.00 \pm \\
41.40\end{array}$ & 0.000 \\
\hline Morphin (mg) & 0 & $15.19 \pm 3.61$ & 0.000 \\
\hline
\end{tabular}

\section{Discussion}

TAP block is a technique of regional anaesthesia by diffusion, which has proven its efficacy in the postoperative multimodal analgesic management of infra-umbilical laparotomies. In a bit to improve on the quality of postoperative pain after total abdominal hysterectomies in resource-limited settings, this study was carried out to evaluate the feasibility of the TAP block and the efficacy of its postoperative analgesic effects. A blind TAP block was performed on 45 patients in Group A. The mean time to onset of sensory block observed in our study was close to those described in the literature $[2,3,4,7]$. The success rate our blind TAP block was $95.5 \%$. This implies that the blind TAP block remains a reliable procedure, especially when performed by a skilled and experienced practitioner.

This success rate is close to the $93.4 \%$ observed by Ndiaye et al. [7] for blind TAP blocks in children undergoing inguinal surgeries [7]. Our success rate is, however, inferior to $100 \%$ reported by Ludot et al. [8], Bhattacharjee et al. [9] respectively for blind TAP blocks in children who underwent appendectomies and adults who underwent total abdominal hysterectomies $[8,9]$. Since the advent of sonography, TAP blocks performed under ultrasound guidance have been widely used to the detriment of the blind technique initially described by Rafi and 
validated by McDonnell [10-13]. However, whether performed under ultrasound guidance or blindly, this technique requires the acquisition of competence whose duration of assimilation is variable with respect to the procedure and the practitioner. Hence, in resource-limited settings, when ultrasound is not available, a blind TAP block should be performed because of its fast onset of action despite the wide interpersonal variability.

With respect to postoperative pain, we have found a superiority of the TAP block in providing immediate postoperative analgesia reflected by lower pain score both at rest and with coughing (Figures $2 \& 3$ ), and the reduction in the consumption of opioid and non-opioid analgesics in patients who received a TAP block (Table 2). The reduction of postoperative pain scores and consumption of analgesics justifies the efficacy of the TAP block, which integrates itself in the multimodal analgesic management of postoperative pain. Studies done by Bhattacharjee et al. [9], Kishore et al. [14], Champaneria et al. [15] reported a reduction in pain scores at rest, associated with a reduction in postoperative consumption of analgesics in patients who had bupivacaine TAP block for total abdominal hysterectomy. Eslamian et al. [16], Soufiene et al. [17] had similar findings after caesarean sections and appendectomies respectively under TAP block.

The transverses abdominis plane block is a relatively new regional anaesthesia technique that provides analgesia to the parietal peritoneum as well as the skin and muscles of the anterior abdominal wall. In agreement with Arbel et al. [18] \& Borendal et al. [19], this block offers a safe, effective and relatively easy mode of analgesia and is effective as part of a multimodal pain management plan, even in resource-constraint areas used by competent practitioners. The limitations of our study lie in the evaluation of pain intensity by visual analogue scale. Indeed, subjectivity in determining the level of the pain by visual analogue scale, which is overestimated and/ or underestimated, probably influenced the postoperative administration of analgesics, either by excess or by default.

\section{Conclusion}

Althoughultrasound guidance is recommended for the success of a TAP block, the blind technique should not be overlooked, particularly in resource-challenged environments. The blind TAP block remains feasible and should be recommended because it integrates itself in the multimodal management strategy of the postoperative pain of total abdominal hysterectomy.

\section{Acknowledgements}

We would like to thank all the study participants for accepting to partake in this study.

\section{Conflict of Interest}

The authors declare that they have no conflicts of interest in relation to this article.

\section{References}

1. Hamitouche Y, Benhamou D (2004) Postoperative pain relief after gynecologic surgery. J Gynecol Obstet Biol reprod 33(1): 7-13.

2. Lemoine A, Vial F, Baka N, Herbain D, Guerci P, et al. (2013) Traversus Abdominis Plane Bloc en chirurgie gynécologique lourde: évaluation des pratiques professionnelles. Ann Fr Anesth Réanim 32: A354-A355.

3. Berger J, Amasse L (2010) TAP bloc: réalisation, indications. Le Praticien en Anesthésie Réanimation 14: 37-42.

4. Petersen PL, Mathiesen O, Torup H, Dahl JB (2010) The transversus abdominis plane block: a valuable option for postoperative analgesia? A topical review. Acta Anaesthesiol Scand 54(5): 529-35.

5. Young MJ, Gorlin AW, Modest VE, Quraishi SA (2012) Clinical Implications of the Transversus Abdominis Plane Block in Adults. Anesthesiol Res Pract 2012: 731645.

6. Aubrun F, Gaulain KN, Fletcher D, Belbchir A, Beloeil H, et al. (2016) Réactualisation de la recommandation sur la douleur postopératoire. Anesth Reanim 2: 421-430.

7. Ndiaye PI, Traore MM, Bah, Fall ML, Leye PA, et al. (2013) Bloc du plan abdominal transverse (TAP block): Etude prospective chez l'enfant au CHU A Le Dantec de Dakar. Revue Africaine d'Anesthésiologie et de Médecine durgence 18(1): 10.

8. Ludot H (2009) Analgesic efficacy of transversus abdominis plane block in children undergoing appendicectomy. Pediatrics 596: 224

9. Bhattacharjee S, Ray M, Ghose T, Maitra S, Layek A (2014) Analgesic efficacy of transversus abdominis plane block in providing effective perioperative analgesia in patients undergoing total abdominal hysterectomy: A randomized controlled trial. J Anaesthesiol Clin Pharmacol 30(3): 391-396.

10. McDonnel JG, Curley G, Carney J, Benton A, Costello J, et al. (2008) The analgesic efficacy of transversus abdominis plane block after cesarian delivery: a randomized controlled trial. Anesth Analg 106(1): 186-191.

11. Carvey J, McDonnell JG, Ochana A, Bhinder R, Laffey JG (2008) The transversus abdominis plane block provides effective postoperative analgesia in patients undergoing total abdominal hysterectomy. Anesth Analg 107(6): 2056-2060.

12. Hériche C, Blot RM, Dubost C (2010). TAP Block: Enfin un bloc de paroi abdominale simple et efficace? Mises au point en Anesthésie Réanimation p. 43-56.

13. Hoarau N (2014) TAP bloc échoguidée pour analgésie après abdominoplastie : une étude randomisée en double insu contre placebo. Etude TAPlastie. Human health and pathology p. 1-75.

14. Kishore H, Raghavan P, Chacko L, Puthur SB, Ancy (2014) Comparision of analgesic efficacy of TAP block with parentral opioid following total abdominal hysterectomy. Journal of evidence based Medicine and Healthcare 1(17): 2121-2128.

15. Champaneria R, Shah L, Geoghegan J, Gupta JK, Daniels JP (2013) Analgesic effectiveness of transversus abdominis plane blocks after hysterectomy: a meta-analysis. European Journal of Obstetrics and Gynecology and Reproductive Biology 166 (1): 1-9.

16. Eslamian L, Jalili Z, Jamal A, Marsoosi V, Movafegh A (2012) Transversus abdominis plane block reduce postoperative pain intensity and analgésic consumption in elective cesarian delivery under general anesthesia. J Anesth 26(3): 334-338.

17. Soufiene S, Marzougui Y, Chebbi N, Kaddou R, Akrout S, et al. (2014) Intérêt du TAP block dans l'analgésie postopératoire au cours de l'appendicectomie en pédiatrie. Ann Fr Anesth Réanim 33S: A238-A239. 
18. Arbel R, Stanleigh J, Loscovich A (2013) Pain management following abdominal hysterectomy: novel approaches and review of literature. Journal of clinical gynecology and Obstetrics 2 (2): 51-55.
19. Borendal Wodlin N, Nilsson L, Kjolhede P (2011) The impact of mode of anaesthesia on postoperative recovery from fast-track abdominal hysterectomy: a randomised clinical trial. BJOG 118(3): 299-308.

\section{Your next submission with Juniper Publishers will reach you the below assets}

- Quality Editorial service

- Swift Peer Review

- Reprints availability

- E-prints Service

- Manuscript Podcast for convenient understanding

- Global attainment for your research

- Manuscript accessibility in different formats ( Pdf, E-pub, Full Text, Audio)

- Unceasing customer service

Track the below URL for one-step submission https://juniperpublishers.com/online-submission.php 
